\title{
Advancing the case for combined laparoscopic-colonoscopic polyp resection?
}

\author{
Aninda Chandra $\cdot$ Abdulzahra Hussain · \\ Tarun Singhal $\cdot$ Biju Aravind $\cdot$ Prakash Sinha
}

Published online: 18 August 2009

(C) Springer Science+Business Media, LLC 2009

\section{Dear Editors:}

Re: Combined laparoscopic-endoscopic resections of colorectal polyps: 10-year experience and follow-up. Wilhelm et al. Surg Endosc (2009) 23:688-693.

This report tries to answer the question regarding the feasibility of laparoscopically assisted resection of difficult "benign" polyps. It would have been useful if the report had contextualized the data presented with regard to the number of patients who underwent alternative/open procedures? The definition of polyps deemed unresectable by colonoscopy is accepted as subjective, although it raises the question whether any screening or repeat colonoscopy was done by an experienced colonoscopist.

Voloyiannis et al. [1] found that selective colonoscopy for 171 of 252 referred patients led to avoidance of resection for 101 patients. Resection was avoided for $58 \%$ of those rescoped and $40 \%$ of all referred. This resection rate may improve with the relatively new technique of colonoscopic (endoscopic) mucosal resection described by Saito et al. [2].

The authors are to be complimented for their honestly appraised complication rate of $25 \%$. The actual percentage probably is higher because the study is retrospective in nature, which leads to difficulties in determining the causes or relevance of reported complications with the procedure. An undeclared percentage of data is being determined by the lead clinician or by patient recall at a later date. Have the authors used a validated scale to classify their complications? Moreover, because intraabdominal abscesses often result from small leaks, do they warrant merely "minor" status? The inclusion of a colonoscopic follow-up evaluation is a useful adjunct, although other trials have used 1-year screening to ensure no polyps were missed. Wade and Braunfeld [3] report a adenoma rate of 58\% at the 1-year colonoscopic follow-up visit, with $9 \%$ of the cases showing adenomas larger than $10 \mathrm{~mm}$, probably representing missed adenomas. In relation to the polyps found at rescoping, did they correlate with previous resections?

Overall, this is an interesting topic highlighted by the authors, and we think this paper is a useful study that could have provided us with more evidence to advance the case for combined laparoscopic-endoscopic resections.

\section{References}

1. Voloyiannis T, Snyder MJ, Bailey RR, Pidala M (2008) Management of the difficult colon polyp referred for resection: resect or rescope? Dis Colon Rectum 51(3):292-295

2. Saito Y, Uraoka T, Matsuda T, Emura F, Ikehara H, Mashimo Y, Kikuchi T, Fu KI, Sano Y, Saito D (2007) Endoscopic treatment of large superficial colorectal tumors: a case series of 200 endoscopic submucosal dissections (with video). Gastrointest.Endosc 66(5): 966-973

3. Waye JD, Braunfeld S (1982) Surveillance intervals after colonoscopic polypectomy. Endoscopy 14(3):79-81

A. Chandra $(\square) \cdot$ A. Hussain $\cdot$ T. Singhal $\cdot$ B. Aravind .

P. Sinha

Department of General Surgery, Princess Royal University

Hospital, Farnborough, London, UK

e-mail: aninda_chandra@hotmail.com 